\title{
Captures
}

Figures, théories et pratiques de l'imaginaire

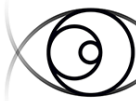

\section{Imaginaire de la ligne. Quand elle joue sur plusieurs tableaux Présentation du dossier}

\section{Véronique Cnockaert}

Volume 2, Number 2, 2017

Imaginaire de la ligne

URI: https://id.erudit.org/iderudit/1059766ar

DOI: https://doi.org/10.7202/1059766ar

See table of contents

Publisher(s)

Figura, Centre de recherche sur le texte et l'imaginaire

ISSN

2371-1930 (digital)

Explore this journal

Cite this article

Cnockaert, V. (2017). Imaginaire de la ligne. Quand elle joue sur plusieurs tableaux : présentation du dossier. Captures, 2(2).

https://doi.org/10.7202/1059766ar
Article abstract

This dossier examines literature, painting, and architecture, as graphical exercises in which lines recompose a world where reality becomes particular and legible. Each work creates its own cartography, yet remains indebted to a graphical imagination in which line, word and idea become one. On top of the lines that surround us emerge the ones engraved in us: they determine our understanding, our comprehension.
C A P T U R E S Figures, théories et pratiques de limaginaire revue interdisciplinaire
This document is protected by copyright law. Use of the services of Erudit (including reproduction) is subject to its terms and conditions, which can be viewed online.

https://apropos.erudit.org/en/users/policy-on-use/ 
Version enrichie de cet article : http://revuecaptures.org/node/1004

\section{Imaginaire de la ligne. Quand elle joue sur plusieurs tableaux}

\section{Présentation du dossier}

\section{Véronique Cnockaert}

Résumé :

Ce numéro propose d'étudier des œuvres littéraires, picturales et architecturales comme autant d'exercices graphiques où la ligne recompose le monde au sein duquel le réel se particularise, devient lisible. Chaque $œ u v r e$ crée ses propres cartographies, mais demeure tributaire d'un imaginaire graphique dans lequel la ligne, le mot et l'idée se rejoignent au point de devenir indiscernables. Au-delà des lignes qui nous entourent, il y a celles qui s'inscrivent en nous et pétrissent notre imaginaire.

This dossier examines literature, painting, and architecture, as graphical exercises in which lines recompose a world where reality becomes particular and legible. Each work creates its own cartography, yet remains indebted to a graphical imagination in which line, word and idea become one. On top of the lines that surround us emerge the ones engraved in us: they determine our understanding, our comprehension.

Un texte littéraire, une peinture, une sculpture ou encore une œuvre architecturale n'est jamais le décalque de la réalité, chaque œuvre crée ses propres cartographies, ses propres espaces-temps, et obéit à l'économie narrative du récit, à l'espace de la toile, à la densité de la matière. L'œuvre est cependant toujours tributaire d'un imaginaire graphique puissant à l'intérieur duquel la ligne, le mot et l'idée s'assemblent, s'unissent, se rejoignent, au point parfois de devenir indiscernables les uns des autres. Qu'il s'agisse de la ligne ascendante ou descendante d'une destinée, des lignes du terrain de jeu, de la ligne morale ou idéologique, de la ligne de vie, de la ligne de tir souvent déviée, des lignes de force ou de faille, des lignes qui découpent l'espace ou de celles qui métaphorisent le temps, toutes, ainsi que l'a admirablement démontré Tim Ingold dans son ouvrage Une brève histoire des lignes, ne cessent d'ordonner l'imaginaire. Aussi le réel est-il graphiquement et culturellement constitué, ordonnancement dont aucune œuvre ne fait l'économie, quelle que soit l'époque de sa création. En effet, notre mémoire ne garde pas uniquement le récit des événements, des sentiments, des émotions, elle inscrit en elle des directions, des hauts et des bas, des chemins plus ou moins tortueux ou non, plus ou moins cohérents et conscients. Dès lors ils font retour à leur manière dans la façon qu'ont les écrivains d'écrire, les peintres de peindre, les photographes de cibler. Au-delà des lignes phénoménales qui nous entourent et forment le cadre de nos espaces, il y a en effet toutes celles qui s'inscrivent en nous et hors de nous, présences abstraites qui pétrissent notre imaginaire et s'actualisent dans l'œuvre. Et si on ne peut avec certitude expliquer dans une œuvre ce qui façonne la ou les lignes, on peut en saisir la volonté, les conséquences et les échappées contrôlées (ou non). 
Qu'il s'agisse de pliures, de pointillés, de courbes, de coupures, de frontières, de démarcations géographique ou sociale, de hauteur ou de chute, sans cesse des lignes s'entrecroisent ou s'excluent, mais toujours tricotent des armatures particulières, métaphoriques, métonymiques ou réelles et dont nos corps font l'expérience par mouvements, sensations, injonctions, etc. D'une manière ou d'une autre, sur ou entre elles, impossible d'échapper aux lignes. Aliénation nécessaire qui donne aux choses une consistance visuelle ou abstraite, et dont les lignes d'horizon et d'écriture sont certainement les exemples les plus probants. Qu'on le veuille ou non, l'imaginaire linéarise le monde dont les battements rappellent le heurté du sismographe ou de l'électrocardiogramme : vie et ligne ne font qu'un.

« Ligne droite et ligne arabesque, intention et expression, roideur de la volonté et sinuosité du verbe, unité du but, variété des moyens, amalgame tout-puissant et invisible du génie, quelle analyse aura le délectable courage de vous diviser et de vous séparer » (151) écrit Baudelaire dans "Le Thyrse » (Petits Poèmes en prose). Sans vouloir les séparer, nous pouvons dire que ce numéro s'arme de ce courage, en prenant en compte et en analysant à partir d'une étude des lignes littéraires, picturales et architecturales, les événements, les phénomènes (géographique, culturel, spirituel, politique, esthétique, etc.) ou les traces qui sont inféodés à la ligne. C'est un fait que celle-ci crée au sein des œuvres des cadrages esthétiques, imaginaires et symboliques précis, ce que chacun des articles ne cesse de confirmer. Ainsi, le présent dossier considère chacune des œuvres analysées comme un exercice graphique au sens fort du terme, où la ligne joue sur tous les tableaux parce qu'elle valorise de facto des plans singuliers de la réalité et recompose de la sorte le monde au sein duquel le réel se particularise et devient lisible. « La géométrie c'est l'art de raisonner juste sur des figures fausses » nous rappelle l'architecte Rudy Ricciotti. Mensonge nécessaire qui nous permet d'appréhender le réel par les lignes, sillons imaginaires de l'espace qui offrent les moyens de consentir plus facilement au ballet vertigineux des circonstances de la vie.

Je tiens ici à remercier chaleureusement Elaine Després et Sébastien Roldan, pour leur vigilance, leurs remarques constructives et le temps qu'ils ont donné à l'élaboration de ce numéro qui leur doit beaucoup.

\section{Bibliographie}

INGOLD, Tim. 2011. Une brève histoire des lignes, traduit par Sophie Renaut. Bruxelles : Zones sensibles, $256 \mathrm{p}$ 